\title{
Measurement of vircator ultra-short solitary electromagnetic pulses
}

\author{
MILOSLAV STEINBAUER, PETR DREXLER, PAVEL FIALA \\ Department of Theoretical and Experimental Electrical Engineering \\ Faculty of Electrical Engineering and Communication, Brno University of Technology \\ Kolejní 4, 61200 Brno \\ CZECH REPUBLIC
}

\begin{abstract}
In connection with the events of the last few years and with the increased number of terrorist activities, the problem of identification and measurement of electromagnetic weapons or other systems impact occurred. Among these are also microwave sources, which can reach extensive peak power of up to $P_{\max }=250 \mathrm{MW}$.

Solitary, in some cases several times repeated, impulses lasting from $t_{\mathrm{p}} \in<1,60>\mathrm{ns}$, cause the destruction of semiconductor junctions. These days we can find scarcely no human activity, where semiconductor structures are not used. The problem of security support of the air traffic, transportation, computer nets, banks, national strategic data centers, and other applications crops up. Several types of system protection from the ultra-short electromagnetic pulses present itself, passive and active protection.

The analysis of the possible measuring methods, convenient for the identification and measurement of the ultrashort solitary electromagnetic pulses is presented in this paper; some of the methods were chosen and used for practical measurement.
\end{abstract}

Keywords: Ultra-short electromagnetic puls, high power microwave generator, calorimetric method, magneto-optical effect, multilayer sensor, vircator, semiconductors.

\section{Methods}

Requirements imposed on the methods, available for capture and measurement of solitary impulses, are very rigorous. The common method applied to the measurement of the magnetic flux density respective power are not applicable for our purpose with regard to the expected impulse's very short length and substantial power Fig. 1. Basic overview of the methods suitable to the purpose named is set out in works $[1,2,3,4,5]$.

\subsection{Methods based on Faraday's induction law}

One group of methods is based on the Faraday's induction law application, where the impulse is located by sensor (coil with $N_{\mathrm{s}}=1 \div 50$ turns). Signal induced in the coil is led to the recording device, generally an oscilloscope. Due to safety requirements, the distance between the sensor and the oscilloscope is $l=50 \mathrm{~m}$. This parameter introduces a quality decrease of the recorded information in the way of the signal amplitude reduction, change of the signal phase and the impulse prolongation.

Elimination of this limitation is in Version I, depicted in Fig. 2, made by backward correction exploiting the Laplace transformation. Impulses up to limit pulse length $T_{\max }=1 \mathrm{~ns}$ were measured by this method and magnetic flux $\phi$ was evaluated [6].
Version II exploits the possibility of principal elimination of the influence of the transmitting line between the sensor and the measuring device by an analogue U/f. converter. Available measuring devices can achieve measured impulses with the limit length $T_{\max }=5 \mathrm{~ns}$.

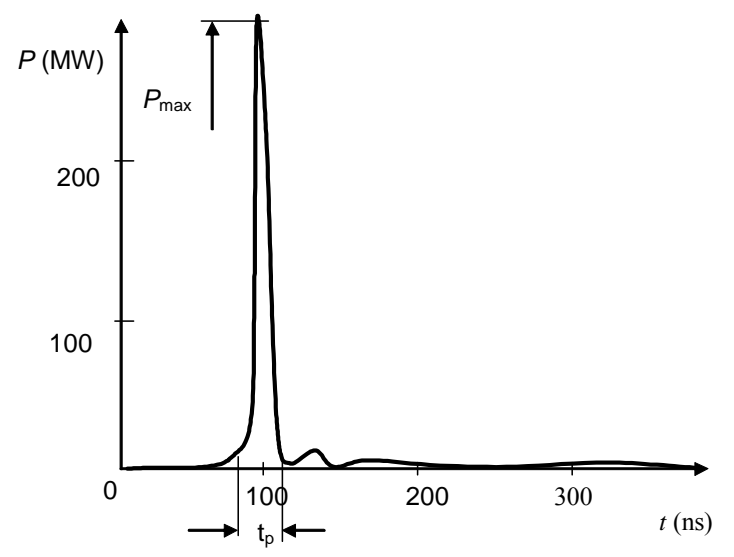

Figure 1. Typical course of short impulse instantaneous power

The solution in Version III is similar to Version II, the difference is in the digital converter applied. By an available measuring devices application and fulfilled sampling theorem we can measure impulses with the limit length $T_{\max }=20 \mathrm{~ns}$. 


\subsection{Method based on Faraday's electro-optic effect}

Version IV in Fig. 2 is based on Faraday's electrooptic effect [4]. Connection between the sensor and the measuring device is implemented in the optical wavelength. The possible active sensors are of three basic types.

The first type is garnet with high Verdet constant, the second is optic fibre, and the third is based on properties of thin mono, multi ferromagnetic nano layers.

Next types of the Version IV sensors are based on the magneto-optic Kerr's effects (MOKE), or surface MOKE (SMOKE) effect. By an available measuring devices application we can measure impulses with the limit length $T_{\max }=0.1 \mathrm{~ns}$.
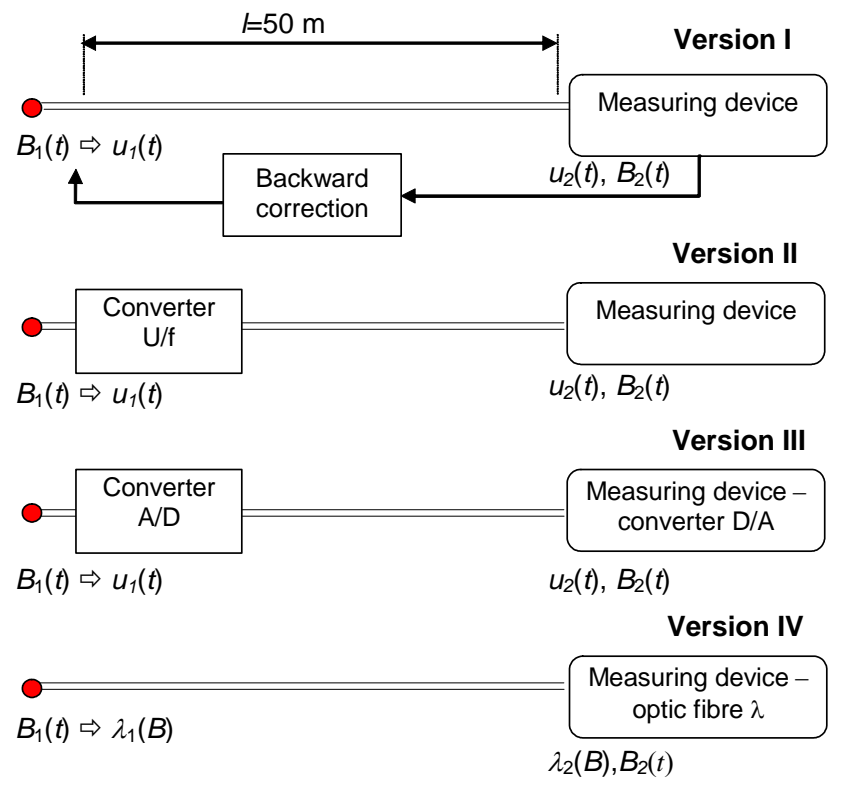

Figure 2. Principles of the methods based on Faraday's induction law and electro-optic effect

The named methods indicate electromagnetic parts of the wave - electric or magnetic. They don't express the power conditions of the electromagnetic wave. For some of the measurement it is essential to evaluate power flow through the defined area.

\subsection{Calorimetric methods}

Into next type of converters belongs the group of calorimetric methods. We can measure power supplied by impulse (Poynting's vector) when we use the calorimetric converter. The sensor is connected to the measuring device (oscilloscope) by an optic fiber of $l=50 \mathrm{~m}$ length. Fig. 3 depicts four versions of the method utilizing calorimetric measurement.

Version I discussed in $[8,9]$ has sensor in the form of an ideal resistor and enables measurement of the maximum value of microwave power $P_{\max }$. The analyzed peak voltage corresponds to peak value of power $P_{\max }$. For available measuring devices we can measure impulses with the limit length $T_{\max }=50 \mathrm{ps}$.

Version II scans change of resistance of the sensor, created by an evaporated thin layer, in dependence on the impulse energy. For available measuring devices we can reach accuracy $30 \%$ up to impulse limit length $T_{\max }=0.1 \mathrm{~ns}$.

Version III is based on the measurement of the temperature change of the thermistor, placed in contact with the layer. Under the same conditions as for previous version we can reach the accuracy improvement of an order of magnitude.

Version IV is the bridge connection of version III. Several (8) thermistors are attached in series to the evaporated layer; next three resistors create a DC bridge of Weston type with the thermistors. The change of the resistance in the thermistor arm is evaluated. The voltage in measuring bridge diagonal is consequently integrated. So the value of the impulse energy is obtained which the measuring device records. For available measuring devices we can measure impulses with the limit length $T_{\max }=0.03 \mathrm{~ns}$ with accuracy to $10 \%$.
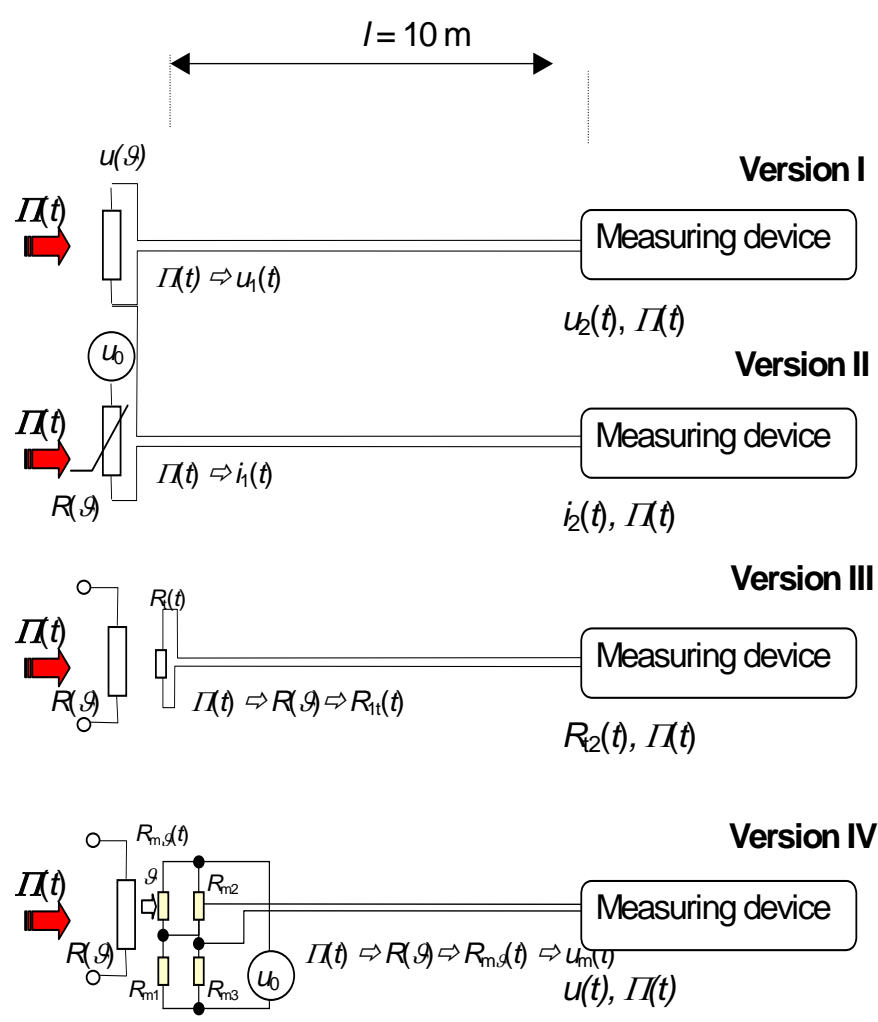

Figure 3. Calorimetric measurement of the solitary impulse power

\section{Experimental realization of the calorimetric method}

The calorimetric sensor was of the disc design. The carbon with changed crystal lattice was used as one of 
the thin layer types. For microwave vircator with output power of $P_{\max }=250 \mathrm{MW}$, length of pulse $t_{\mathrm{p}} \in<10$, $60>$ ns combined calorimetric sensors were designed as is shown in Fig. 3. - version IV and version I. The concept was designed after consultation [10] for supposed power and the impulse length with room for absorption and damping of the possible back EMG wave.
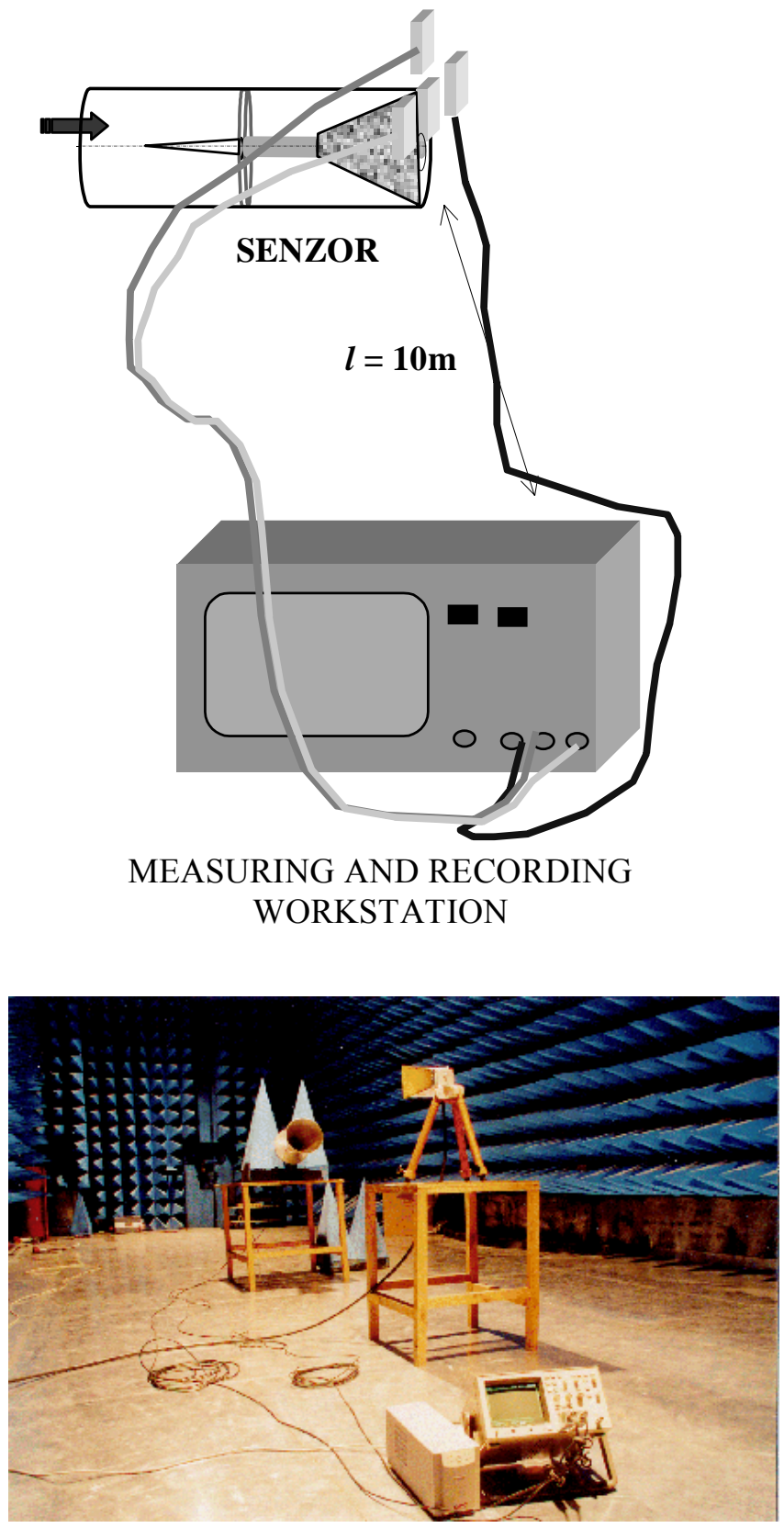

Figure 4. Measuring workstation

The length of connection between sensor and measuring device was $l_{\min }=10 \mathrm{~m}$ due to the safety requirements. When the vircator is in function, the hard RTG emission is generated beside the microwave emission. The energy of electron beam is $W_{\mathrm{b}}=1 \mathrm{MeV}$. The course of the input voltage in the calorimetric bridge for incident energy $W_{\mathrm{em}}=10 \mathrm{~J}$ is shown in Fig. 5., Fig. 7. The courses of the reference voltage and the pulse energy $W_{\mathrm{p}}$ measured are in Fig. 6, Fig. 8.

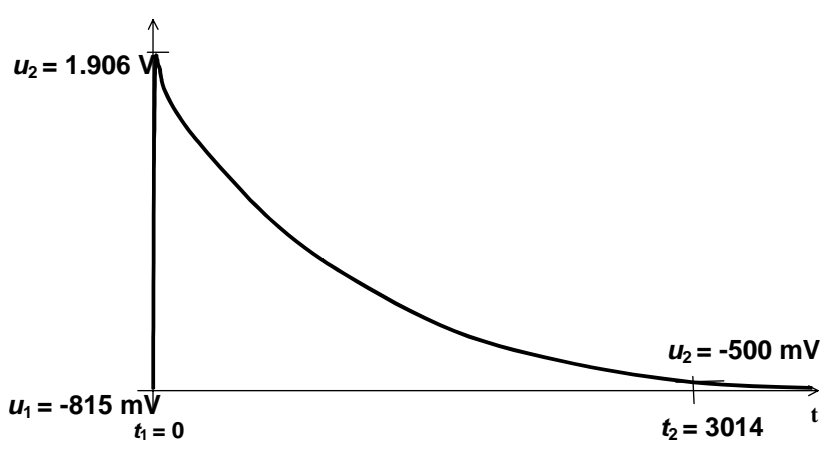

Figure 5. Course of measured voltage in the calorimeter bridge output

\section{CONCLUSION}

The overview of several methods suitable for measurement of the short solitary impulses with high power level was given. The characteristics of the designed method were discussed. Some methods were experimentally tested and evaluated. In the Fig. 9 is shown microwave pulsed power generator based on the virtual cathod effect - vircator.

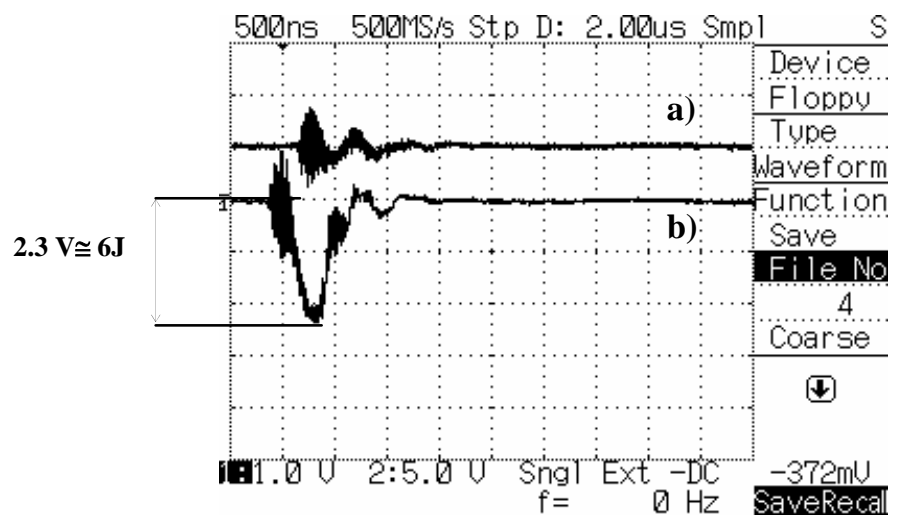

Figure 6. Vircator - measurement on the calorimeter integrator: a) reference voltage, b) energy $W_{\mathrm{p}}$ value with high jamming.

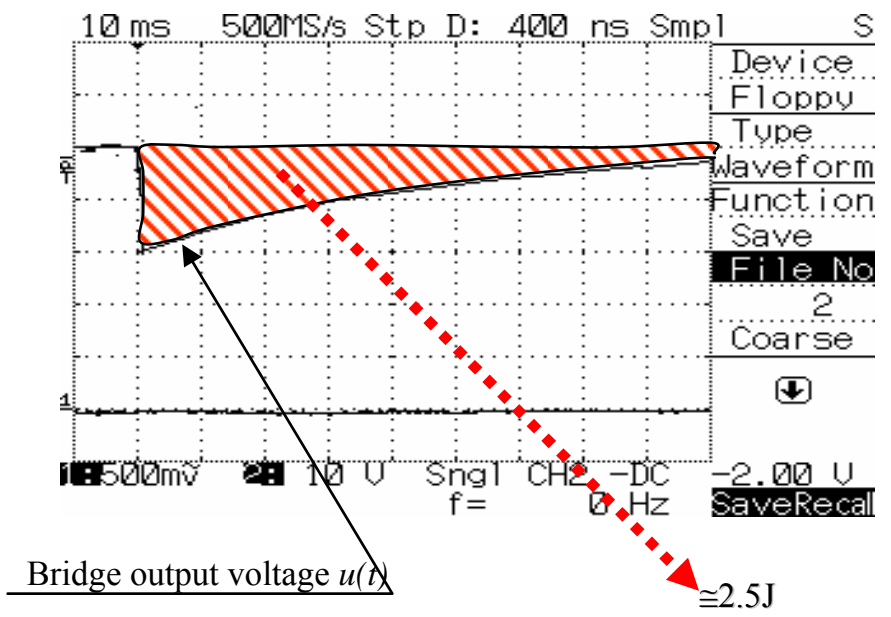

Figure 7. Measured voltage in the calorimeter bridge output 


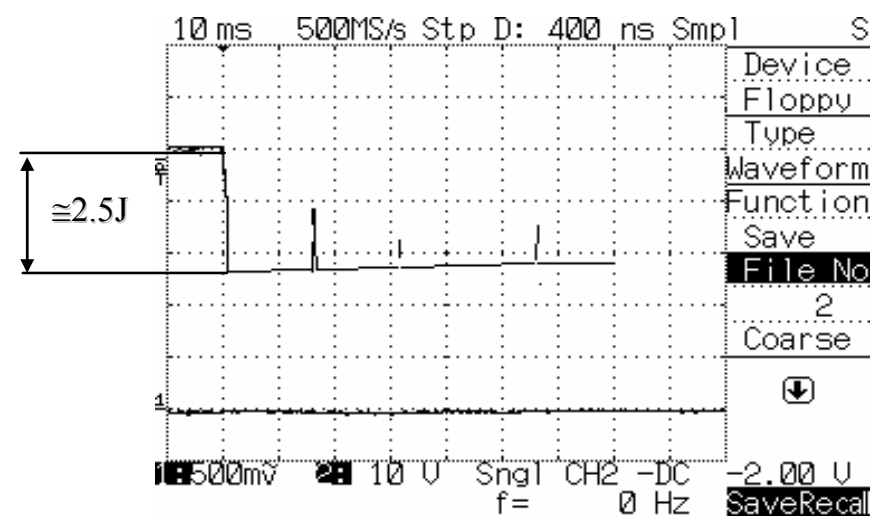

Figure 8. Vircator - measurement on the calorimeter integrator energy $W_{\mathrm{p}}$ value

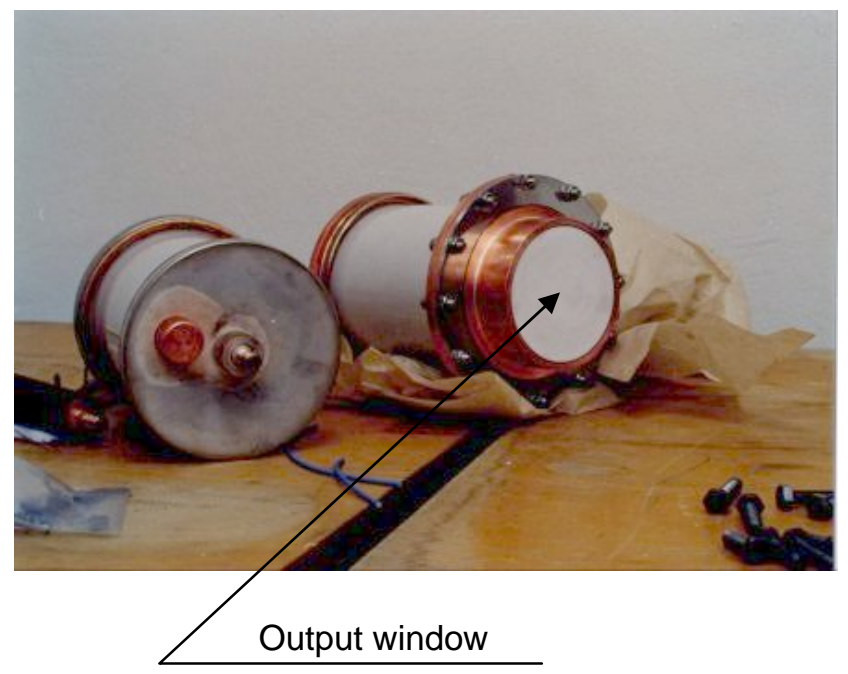

Figure 9. Vircator $-P_{\max }=250 \mathrm{MW}$

Figure 10. Calorimetric sensor for closed space measurement - waveguide

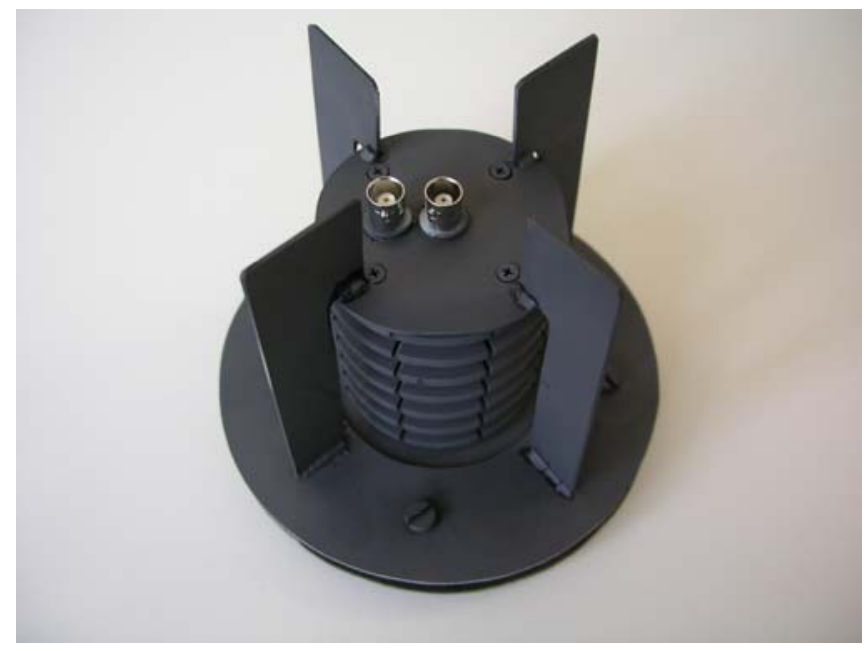

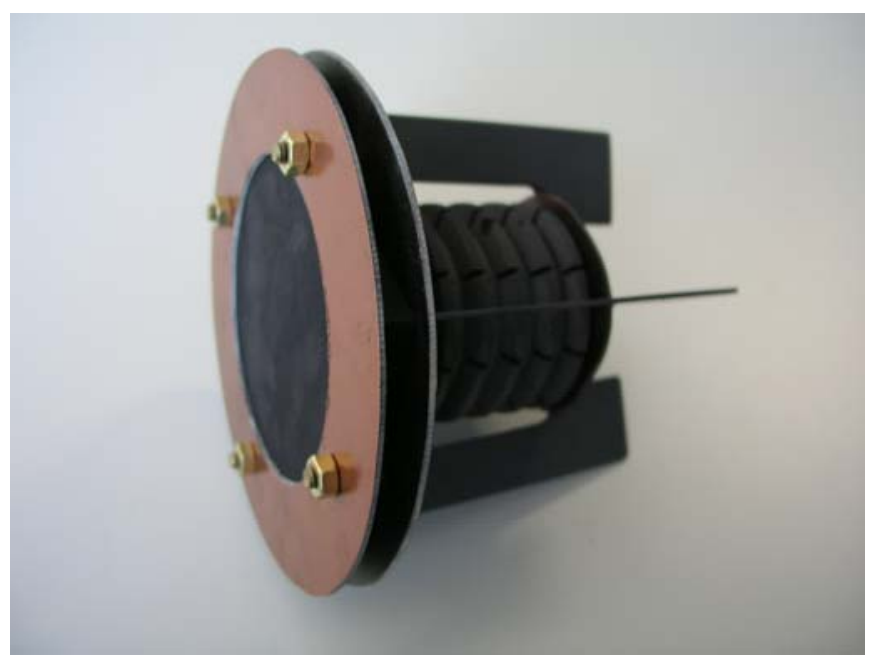

Figure 11. Calorimetric sensor for free space measurement

\section{References:}

[1] H.Ott, Noise reduction techniques in electronic systems. John Wiley \&Sons, 1988, ISBN0-47185068-3.

[2] J.H.Booske et al., Studies of non-thermal effects during intense microwave heating of crystalline solids, Proc. 3-rd Symposium Microwave processing of materials. Vol. 269, pp. 137-143, Pittsburgh 1992.

[3] R.J.Barker, High-Power Microwave Sources and Technologies. IEEE, 2001; ISBN 0-7803-6006-0.

[4] J. A. Riordan; F.G. Sun; Z.G.Lu; X. C. Zhang, Free-space transient magneto-optic sampling," Applied physic letters 1997, Vol 71, Iss 11, pp 1452-1454. ISSN:0003-6951.

[5] P.Ripka, Magnetic sensors and magnetometers. Artech House, London, 2001, ISBN 1-58053-0575.

[6] P. Fiala, Non-conventional sources of electrical energy, VUT FEKT v Brně, June 2003, Czech Republic.

[7] R. J. Barker, E. Schamiloglu, High -Power microwave sources and technologies, IEEE Press, 2001.

[8] M. Kasal, Multinuclear measuring channel of NMR spectrometer. FE VUT v Brně 1984, in Czech, Czech Republic.

[9] M. Pfeffer, M. KASAL, Automatic impuls reflectometer, (Automatický impulsní reflektometr), Sdělovací technika, 1986, č.10/11, ss. 380-383, in Czech, Czech Republic

[10] Šunka,P.: Verbal information, UFP AV ČR, Praha, 6.2003, 11.2003, www.ipp.cas.cz. 